\title{
Use of Intravenous Immunoglobulin in the Treatment of Severe COVID-19 Disease - A Case Series
}

\author{
Arun Agarwal, Rekha Jakhar, Ambika Sharma and Aakanksha Agarwal
}

\section{ABSTRACT}

Objective: To study and document the outcomes of adjuvant use of high dose intravenous immunoglobulin (IVIg) therapy in patients with severe or critical corona virus disease 2019 (COVID-19). We report in a case series of five patients who were admitted with severe and critical COVID-19 disease and were treated with adjuvant IVIG along-with the institute's standard of care (SOC) treatment.

Methods: It is a retrospective observational study. We retrospectively collected data on all patients with COVID-19 disease who were hospitalized in author's unit. The severe and critical disease patients who received IVIg were shortlisted and are discussed.

Results: Data from 101 patients were analyzed. Of them 5 patients were treated with IVIG along with institution's SOC. 4 patients were male and 1 was female. Except one patient $(P 2)$ all were above 60 years of age and all had one or more co morbidities with Diabetes mellitus (DM) and Hypertension (HT) present all of them. 3 patients had past history of pulmonary tuberculosis (P1, P4 and P5). P2 had chronic kidney disease (CKD) and $\mathrm{P} 4$ had coronary artery disease (CAD) with cardiac resynchronization therapy (CRT) device in situ. Median length of stay was 13 days and 4 of them were discharged.

Conclusions: This small case series demonstrates that administration of IVIg in patients with severe COVID-19 disease, who did not respond to usual standard of care treatment, could improve clinical outcome and reduce mortality rate. It should be especially considered in cases with severe critical COVID-19 disease along with evidence of hyper inflammation /cytokine storm. Clinical efficacy is possibly driven by its anti-cytokine effects, reduction of inflammation by inhibition of complement activation, and down-regulation of $B$ and $T$ cells' functions. Among the various inflammatory markers IVIg reduced CRP and D Dimer levels. It did not show relevant effect on other inflammation markers. However, multicenter studies with large sample size are needed to substantiate these observations.

Submitted: September 13, 2021

Published: October 27, 2021

ISSN: 2593-8339

DOI: $10.24018 /$ ejmed.2021.3.5.1061

\section{A. Agarwal*}

Department of Internal Medicine, Fortis Escort Hospital, Jaipur, Rajasthan, India.

(e-mail: mpicdrarun@gmail.com)

R. Jakhar

Department of Internal medicine, Fortis Escort Hospital, Jaipur, Rajasthan, India.

(e-mail: sdbrekhajakhar@gmail.com)

A. Sharma

Department of Internal medicine, Fortis Escort Hospital, Jaipur, Rajasthan, India.

(e-mail:

ambikasharma305@gmail.com)

A. Agarwal

Department of Radiodiagnosis, All India Institute of Medical Sciences Rishikesh, India.

(e-mail: a.agarwal.1992@gmail.com)

*Corresponding Author

Keywords: COVID-19, cytokine storm, immunotherapy, IVIg, SARS-CoV2.

\section{INTRODUCTION}

Towards the end of 2019, in the city of Wuhan, China, few cases of pneumonia were identified to be caused by a novel corona virus. The culprit virus was highly communicable, and it rapidly spread all over the world, resulting in a pandemic as declared by the World Health Organization on March 11th, 2020. Earlier, in February 2020, the World Health Organization designated the disease as COVID-19, which stands for corona virus disease 2019. The culprit virus (i.e., 2019-nCoV) that causes COVID-19 was designated severe acute respiratory syndrome corona virus 2 (SARS-CoV-2).

With rapid dissemination of COVID-19, the understanding of the pathogenesis of the disease and patient management has continuously evolved over time. Nevertheless, since emergence of COVID-19 pandemic, not much specific and targeted pharmacological treatments are available yet. Present COVID-19 therapeutics, Remdesivir, an antiviral agent, is the only Food and Drug Administration (FDA) approved drug for the treatment of COVID-19. Besides Remdesivir, management of COVID-19 includes various pharmacologic interventions authorized under emergency use authorization (EUA) by FDA and includes anti SARS-COV2 antibody products (convalescent plasma, SARS-COV-2 specific immunoglobulin's, sotrovimab, bamlanivimab plus Etesevemab or the casirivimab plus imdevimab combination), immunomodulation (corticosteroids, baricitinib+Remdesivir, interleukin (IL)-1 inhibitor Anakinra, Interferon beta), other immunotherapy's (blocking antibodies to IL-6 receptor) and intravenous immunoglobulin have provided encouraging results [1]. Cell based therapy is still under evaluation. 
Clinical presentation of COVID-19 is variable, and the spectrum of illness can range from asymptomatic infection to mild, severe, or critical disease-causing pneumonia with acute respiratory distress syndrome (ARDS) and even death. Severe COVID-19 patients who suffer from ARDS and multi-organ dysfunction have high mortality. Several studies have shown that this is closely related to the cytokine storm or cytokine release syndrome (CRS) and it appears to be the major underlying cause of morbidity and mortality [1],[2]. Cytokine release syndrome (CRS) is a systemic hyper inflammatory condition due to unregulated host immune response presenting secondary to monocyte, macrophage and dendritic cell activation in severe COVID-19 infection and has been implicated in disease pathophysiology [3].

There are several case reports, case series, and a few studies from China that critically ill COVID-19 patients may benefit from treatment with IVIg [4]-[11]. In the United States, the FDA is also facilitating the evaluation of hyper immune globulin for patients with COVID-19 [12]. The indications, outcomes, and effect(s) of IVIg on inflammatory markers are discussed.

\section{METHODS}

The present study has been approved by the institutional Ethics committee (IEC) vide letter No. FEHJ/IEC/20/19 dated $28 / 12 / 2020$. It is an observational retrospective study. The aim was to review the limited number of cases of severe critical COVID-19 disease patients who were admitted in authors unit and treated with IVIg along with institution's SOC and to observe its effects on the clinical outcomes and inflammatory markers.

All patients who presented to flu clinic with influenza like illness (ILI) at Fortis escorts Hospital, Jaipur, India, were tested for COVID-19 by real-time reverse transcription polymerase chain reaction (rRT-PCR) from upper respiratory samples including both nasopharyngeal and oropharyngeal swabs and those with moderate, severe or critical respiratory illness were admitted to COVID-19 unit for further evaluation and management [13]. Patients with mild disease were advised home treatment and were excluded from the study. Medical records of such patients admitted between $01 / 08 / 2020$ to $31 / 12 / 2020$ were extracted from medical records department of our institute.

Various epidemiological and laboratory parameters including age, gender, history of smoking, presenting complaints, vital parameters, co-morbidities, Coinfection, complete blood counts (CBC), Neutrophil Lymphocyte ratio (N/L ratio), inflammatory markers, biochemistry, High resolution Computerized Tomography of chest (HRCT) and $\mathrm{X}$ ray chest, presence of cytokine storm, Intensive care unit (ICU) admission, type of oxygen support, treatment details, length of hospital stay, and mortality in the patients who received IVIg were recorded and tabulated. After obtaining informed consent from patient and/or attendants, IVIg was administered in a dose of $0.4 \mathrm{~g} / \mathrm{kg}$ for 5 days as per hospital protocol. In all these 5 patients IVIG was initiated within 7 days of hospital admission. The detail profile of these 5 patients (P1 to P5) is presented in Table I.

\section{RESUlts AND FINDINGS}

Of total 101 patients with COVID-19 infection admitted during the study period, 5 patients were treated with IVIg. None of these 5 patients had history of smoking. Male female ratio was $4: 1$.

As regards the results and findings of these 5 patients presented, following patterns were observed:

1. Comorbidities: Co morbidities and other conditions that were associated with severe critical COVID-19 illness included cardiovascular disease (1 patient), Hypertension (5 patients), Diabetes mellitus (5 patients), chronic kidney disease (1 patient), and history of pulmonary tuberculosis (3 patients). One of them had CRT in situ.

2. Co-infection: Only one patient, P3 was found to have IgM antibodies to respiratory synctitial virus (RSV) suggesting recent RSV infection. RT-PCR for respiratory viruses was negative in all 5 patients.

3. Symptoms and critically ill patients: All 5 patients had symptoms of cough. Fever and were critically ill. Shortness of breath (dyspnea) was present in 2 patients. P1was 62 years male patient and was referred from outside with COVID-19 positive status and acute respiratory distress syndrome (ARDS). He was mechanically ventilated but developed catastrophic COVID-19 infection with severe acute acrocyanosis and digital gangrene. He subsequently succumbed to his disease due to multi organ dysfunction [14]. P4 was 72 years male patient with moderate Left ventricle systolic dysfunction, CRT device in situ and a past history of pulmonary tuberculosis. His hypoxemic respiratory failure was managed with escalation of oxygen therapy i.e., High flow nasal cannula (HFNC) along with oxygen via venture mask. He was discharged after 14 days of hospital stay. P2, P3, and P5 were managed with HFNC.

4. Radiological imaging: All 5 patients were evaluated by HRCT of the chest which demonstrated typical ground-glass opacity and viral pneumonia findings in all of them. Severe disease with computer tomography severity score (CTSS) > 15 on HRCT was seen in P2, P3 and P4 and moderate disease in $\mathrm{P} 1$ and $\mathrm{P} 5$ respectively [15]. Chest $\mathrm{X}$-ray was performed in all 5 patients and show bilateral reticular shadowing or bilateral diffuse infiltration. Fig. 1 show axial image of HRCT before and after IVIg treatment in these patients. Fig. 2 shows the X Ray Chest before, during and after the IVIg treatment.

5. Laboratory findings: All 5 patients show Lymphopenia. The highest N/L ratio of 46.5 was seen in P3. CRP $>10$ times the upper limit of normal (ULN) was seen in all 5 patients. The maximum was $228 \mathrm{mg} / \mathrm{L}$ in P2. D-Dimer levels were significantly raised and were $>10$ times of ULN in P1, P3, P4, and P5 with $11140 \mathrm{ng} / \mathrm{ml}$ in P3. Serum Ferritin levels were raised significantly in P1, P2, P3 and P4 with levels above $>100$ times of ULN in P1 and P2. IL-6 levels were also raised in all 5 patients. All these patients were in cytokine storm with evidence of ARDS. Fig. 3 show serial values of CRP during IVIg treatment and Fig. 4 show trend in D Dimer values during IVIg treatment. 
European Journal of Medical and Health Sciences www.ejmed.org

TABLE I: Clinical, LABORATORY, IMAging, TREATMENT Given AND Other SALIENT FEATURES OF PATIENTS TREATEd With IVIG.

\begin{tabular}{|c|c|c|c|c|c|}
\hline Parameter & P1 & $\mathrm{P} 2$ & P3 & $\mathrm{P} 4$ & P5 \\
\hline Gender & Male & Male & Male & Male & Female \\
\hline Age & 62 Years & 45 Years & 63 Years & 73 Years & 72 Years \\
\hline Day of Admission & $26 / 09 / 2020$ & $22 / 10 / 2020$ & $29 / 10 / 2020$ & $02 / 11 / 2020$ & $20 / 11 / 2020$ \\
\hline Co-morbidity & $\begin{array}{c}\text { DM, HTN, old healed } \\
\text { Pulmonary } \\
\text { Tuberculosis }\end{array}$ & $\begin{array}{l}\text { CKD stage } 5, \\
\text { HTN, DM, PDR }\end{array}$ & DM, HTN & $\begin{array}{c}\text { DM, HTN, CAD } \\
\text { LBBB, LV } \\
\text { Dysfunction, CRT IN } \\
\text { situ, old healed } \\
\text { Pulmonary } \\
\text { Tuberculosis }\end{array}$ & $\begin{array}{c}\text { HTN, DM, old healed } \\
\text { Pulmonary } \\
\text { Tuberculosis }\end{array}$ \\
\hline Co-infection & None & None & $\begin{array}{l}\text { RSV IgM antibody } \\
\text { detectd }\end{array}$ & None & None \\
\hline Complications & $\begin{array}{c}\text { Digital } \\
\text { gangrene/Acrocyanosi } \\
\text { s, Atrial fibrillation, } \\
\text { Acute Kidney injury }\end{array}$ & $\begin{array}{c}\text { Maintenance } \\
\text { Hemodialysis, } \\
\text { Bacteremia, Urinary } \\
\text { tract Infection }\end{array}$ & $\begin{array}{l}\text { Atrial fibrillation, } \\
\text { Bacteremia, sepsis }\end{array}$ & & Urinary tract Infection \\
\hline Chief complaints & SOB,Cough,Fever & Fever,Cough & Fever,Cough & SOB,Cough,Fever & Fever,Cough \\
\hline \multicolumn{6}{|c|}{ Lab Findings } \\
\hline $\begin{array}{c}\mathrm{ABG} \\
\left(\mathrm{pH} / \mathrm{pCO} 2 / \mathrm{pO} 2 / \mathrm{HCO}^{-}\right)\end{array}$ & $\begin{array}{c}7.3 / 32.7 / 66.3 / \\
18.4\end{array}$ & $\begin{array}{c}7.48 / 21.8 / 47.6 / \\
16.1\end{array}$ & $\begin{array}{c}7.49 / 53.2 / 71.9 / \\
39.9\end{array}$ & $7.5 / 32 / 47 / 25$ & $\begin{array}{c}7.31 / 47.6 / 76.6 / \\
23.6\end{array}$ \\
\hline $\begin{array}{c}\text { ESR } \\
\left(<20 \mathrm{~mm} / 1^{\mathrm{st}} \mathrm{hr}\right)\end{array}$ & 60 & 95 & 32 & 80 & 82 \\
\hline CRP (<6 mg/litre) & 218.6 & 228 & 61.3 & 130.6 & 60 \\
\hline $\mathrm{LDH}$ & 639 & 1181 & 769.5 & 482 & 407 \\
\hline $\begin{array}{l}\text { D-DIMER } \\
(<500 \mathrm{ng} / \mathrm{ml})\end{array}$ & 7090 & 2420 & 11140 & 6260 & 9250 \\
\hline $\begin{array}{c}\text { FERRITIN } \\
(13-150 \mathrm{ng} / \mathrm{ml})\end{array}$ & $>20000$ & 19522 & 1536 & 1177 & 191.8 \\
\hline $\begin{array}{l}\text { PROCALCITONIN } \\
(<0.046 \mathrm{ng} / \mathrm{ml})\end{array}$ & 1.05 & 78.84 & 0.099 & 0.417 & 0.078 \\
\hline IL-6 $(<7$ pg/ml $)$ & 26.5 & 43.31 & 210.2 & 271.6 & 45.19 \\
\hline $\begin{array}{l}\text { SGOT/SGPT } \\
\text { (Upto } 32 \mathrm{U} / \mathrm{L} \text { ) }\end{array}$ & $33 / 37$ & $75 / 15$ & $34 / 38$ & $45 / 21$ & $35 / 29$ \\
\hline $\mathrm{HB}(12-15 \mathrm{gm} / \mathrm{dl})$ & 16.6 & 10.4 & 16.2 & 14.5 & 11.1 \\
\hline $\begin{array}{c}\text { TLC } \\
\left(4-5 \times 10^{3} / \mathrm{mm} 3\right)\end{array}$ & 27.7 & 10.9 & 22.7 & 11.5 & 10.3 \\
\hline $\begin{array}{c}\text { PLATELETS } \\
\left(150-410 \times 10^{3} / \mathrm{mm} 3\right.\end{array}$ & 227 & 431 & 215 & 307 & 280 \\
\hline $\mathrm{N} / \mathrm{L}(<3.5)$ & 18 & 15.3 & 46.5 & 18 & 18.2 \\
\hline $\begin{array}{l}\text { PRO-BNP } \\
(<125 \mathrm{pg} / \mathrm{ml})\end{array}$ & 1176 & 18400 & 3657 & 767 & 45.5 \\
\hline HRCT CTSS & $12 / 25$ & $20 / 25$ & $17 / 25$ & $16 / 25$ & $9 / 25$ \\
\hline Blood Culture & Sterile & $\begin{array}{l}\text { Brevundimonas } \\
\text { Diminuta }\end{array}$ & Aeromonas salmonicida & Enterococcus faecium & $\begin{array}{l}\text { Staphylococcus } \\
\text { hominis }\end{array}$ \\
\hline $\begin{array}{c}\text { Urine Culture } \\
\text { ET/ Sputum culture }\end{array}$ & $\begin{array}{l}\text { Candida other than } \\
\text { albicans, } \\
\text { Trichosporon asahii }\end{array}$ & Klebsiella Pneumoniae & sterile & $\begin{array}{c}\text { Candida } \\
\text { albicans,enterococcus } \\
\text { faecium } \\
\text { Sputum-candida } \\
\text { albicans } \\
\end{array}$ & $\begin{array}{c}\text { Klebsiella } \\
\text { Pneumoniae, } \\
\text { Escherichia coli. }\end{array}$ \\
\hline \multicolumn{6}{|c|}{ COVID Treatment } \\
\hline Remdesivir & Yes & Yes & Yes & Yes & Yes \\
\hline LMWH/UFH & Yes & Yes & Yes & Yes & Yes \\
\hline CCP $(200 \mathrm{Ml})$ & No & No & No & No & Yes \\
\hline IVIg/Day Of Initiation & Yes /D6 & Yes/D3 & Yes/D5 & Yes / D2 & Yes/D4 \\
\hline Dexamethasone & Yes & Yes & Yes & Yes & Yes \\
\hline Tocilizumab & No & No & Yes & Yes & No \\
\hline $\begin{array}{c}\text { Mode Of Oxygen } \\
\text { Therapy }\end{array}$ & CMV & HFNC & HFNC & $\mathrm{HFNC}+\mathrm{O} 2$ Mask & HFNC \\
\hline LOS & 13 Days & 8 Days & 12 Days & 17 Days & 14 Days \\
\hline Outcome & Death & Discharge & Discharge & Discharge & Discharge \\
\hline
\end{tabular}

Abbreviations: F: Female; M: Male; SOB: Shortness Of Breath; BP: Blood Pressure; HR: Heart Rate; T: Temperature; RR: Respiratory Rate; SPO2:Oxygen Saturation; RSV: Respiratory Synctitial Virus; Igm: Immunoglobulin M; DM: Diabetes Mellitus; HT: Hypertension; CAD: Coronary Artery Disease; LBBB: Left Bundle Branch Block; CRT: Cardiac Resynchronization Therapy; HD: Hemodialysis; GNB: Gram Negative Bacilli; UTI: Urinary Tract Infection; ET: Endotracheal Tube; Hb: Hemoglobin; TLC: Total Leucocyte Count; N/L: Neutrophil Lymphocyte Ratio; AST: Aspartate Aminotransferase; ALT: Alanine Transaminase; ESR: Erythrocyte Sedimentation Rate; CRP: C Reactive Protein; IL-6: Interleukin 6; LDH: Lactate Dehydrogenase; Pro BNP: Pro-B-Type Natriuretic Peptide; HRCT: High Resolution Computed Tomography; CTSS: Computed Tomography Severity Score; LMWH: Low Molecular Weight Heparin; UFH: Unfractionated Heparin; CCP: COVID Convalescent Plasma; ICU: Intensive Care Unit; AEBA: Acute Exacerbation Of Bronchial Asthma; ARDS: Acute Respiratory Distress Syndrome; VAP: Ventilator Associated Pneumonia; LOS: Length of Stay; U/L: Units per Liter; ng/ml: Nanogram per milliliter; pg/ml: Picogram per millilitre; g/dl: Gram per decilitre. 


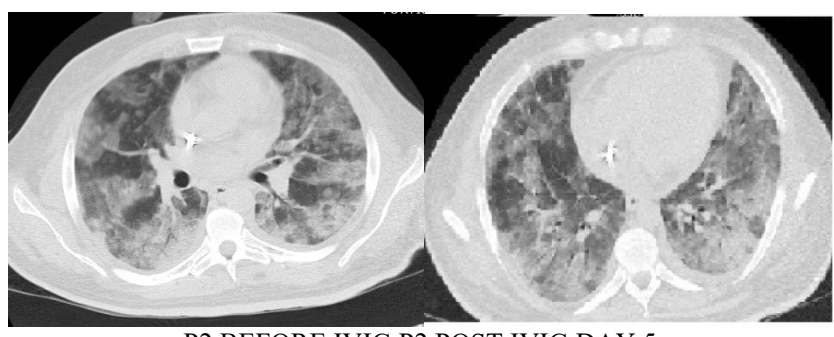

P2 BEFORE IVIG P2 POST IVIG DAY 5

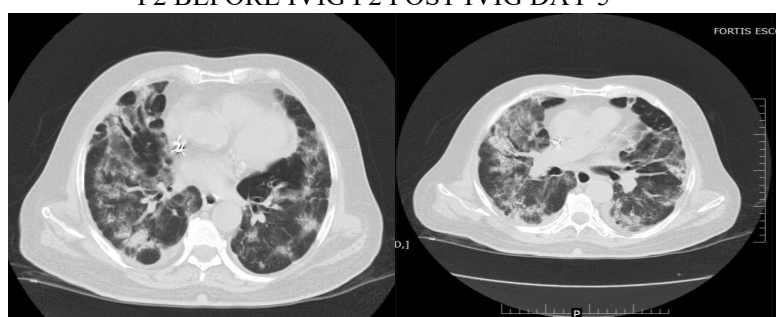

P4 BEFORE IVIG P4 POST IVIG DAY 7

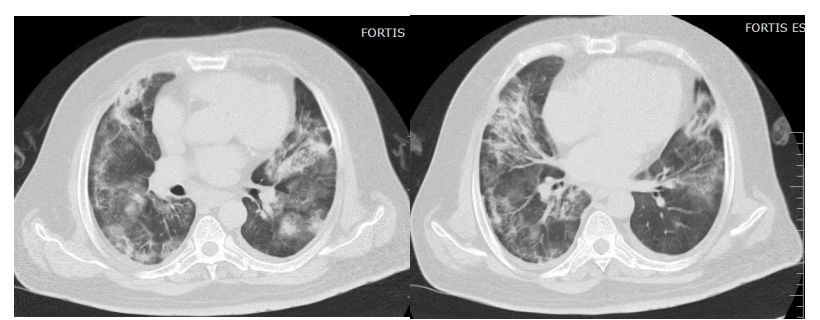

P3 BEFORE IVIG P3 POST IVIG DAY 7

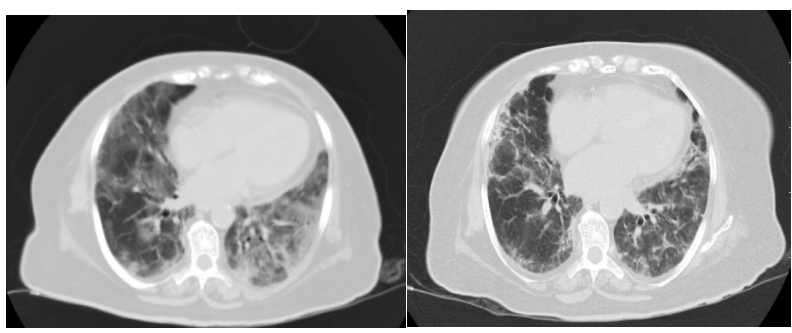

P5 BEFORE IVIG P5 POST IVIG DAY 10

Fig. 1. Axial HRCT views of patients before and after IVIg treatment.

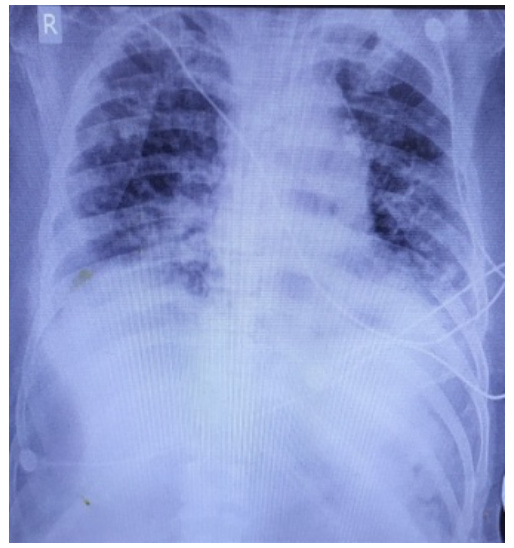

P1 X-RAY CHEST DAY 0
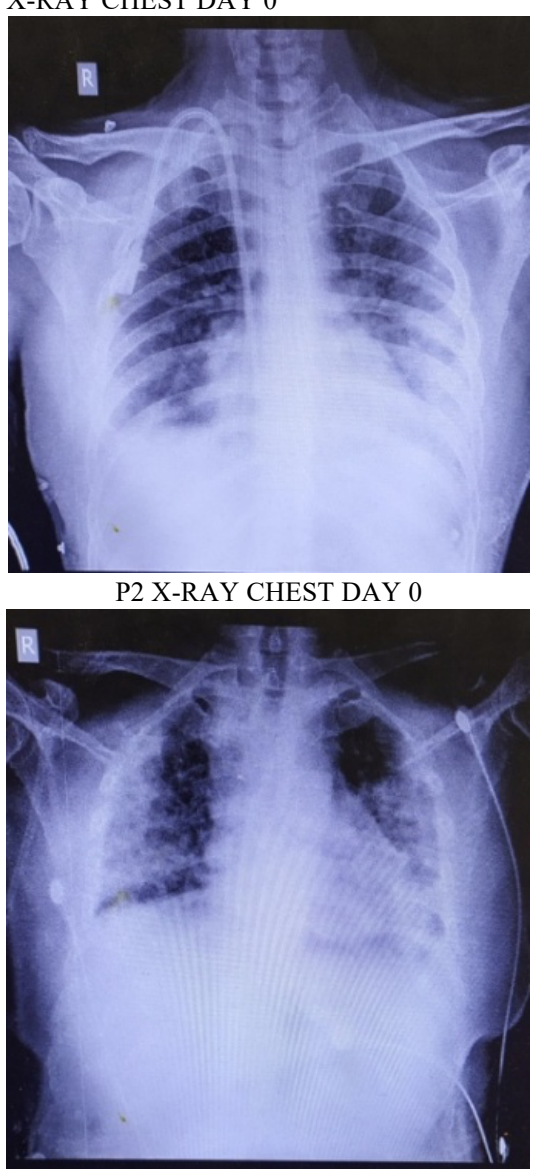

P3 X-RAY CHEST DAY 0

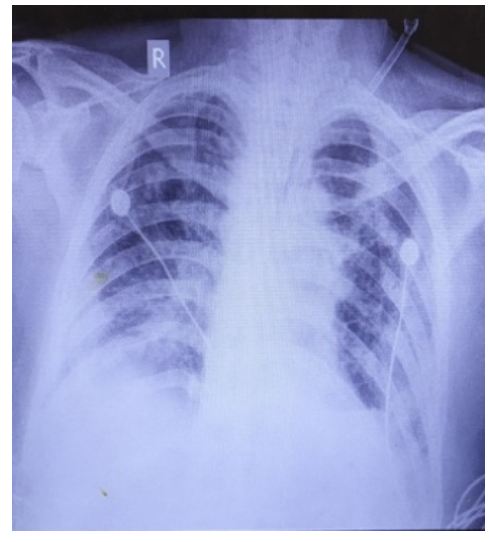

P1 X-RAY CHEST DAY 3

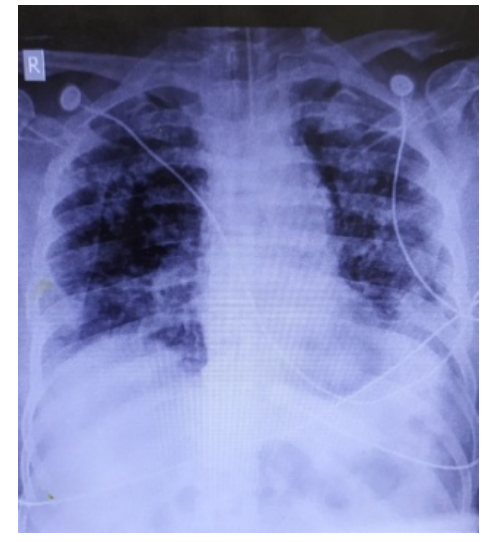

P1 X-RAY CHEST DAY 6

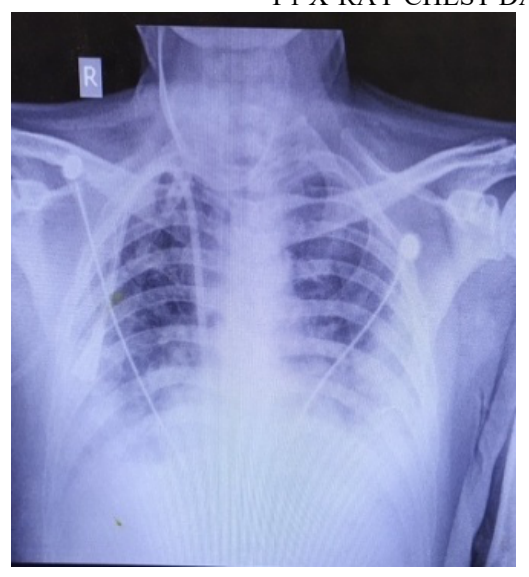

P2 X-RAY CHEST DAY 3

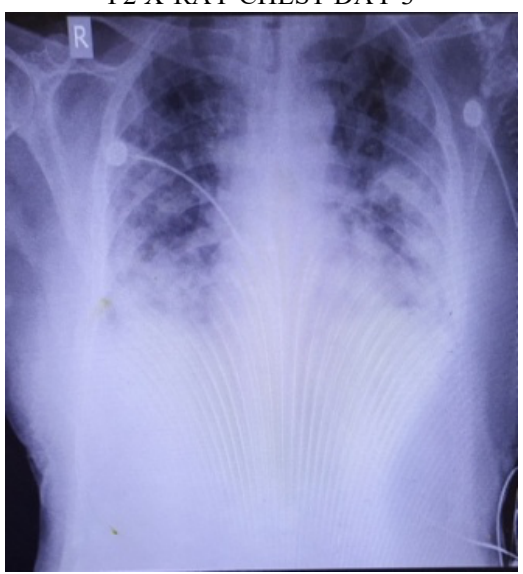

P3 X-RAYR CHEST DAY 8 


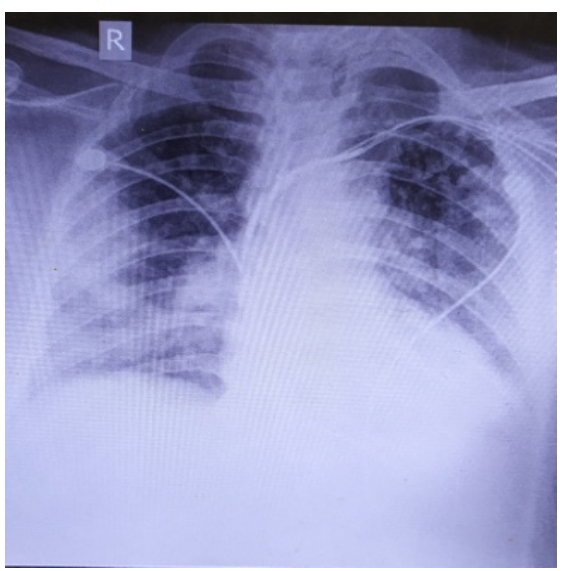

P4 X-RAY CHEST DAY 1

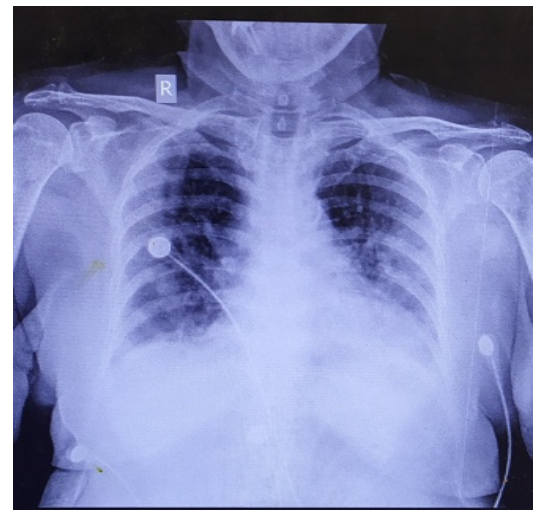

P5 X-RAY CHEST DAY 1

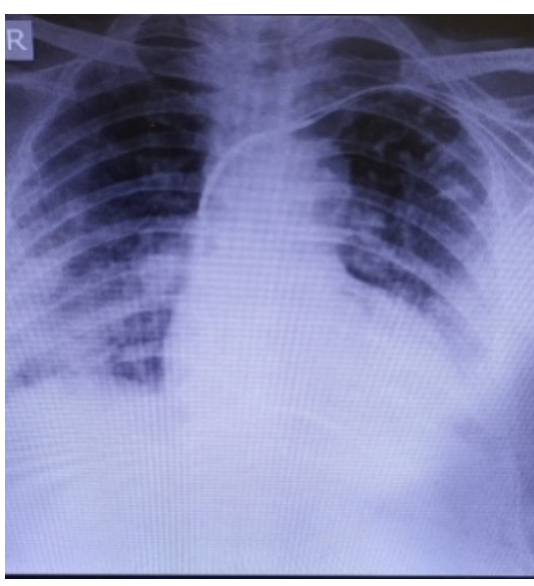

P4 X-RAY CHEST DAY 3

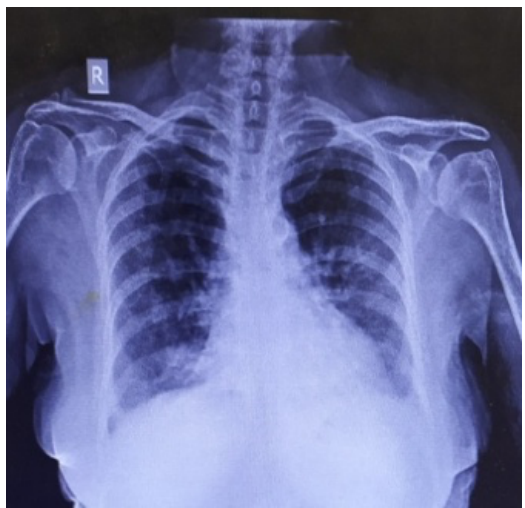

P5 X-RAY CHEST DAY 3

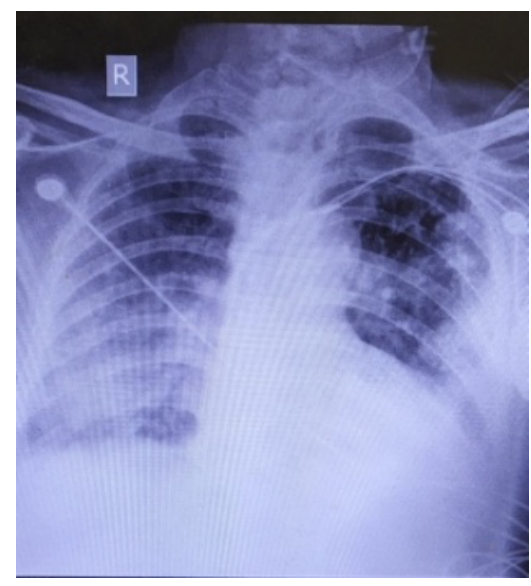

P4 X-RAY CHEST DAY 8

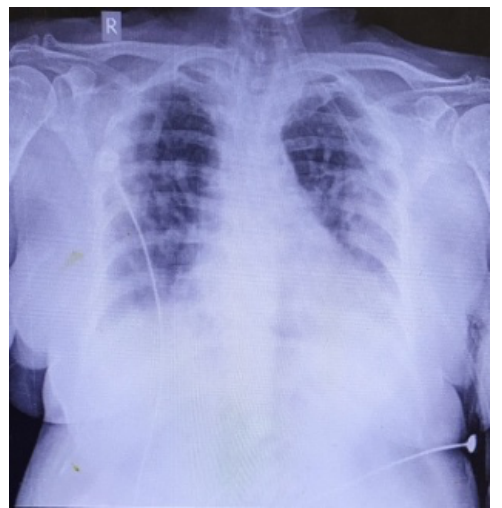

P5 X-RAY CHEST DAY 9

Fig. 2. X-ray chest of patients on day 0 or 1 , during and after treatment with IVIg.

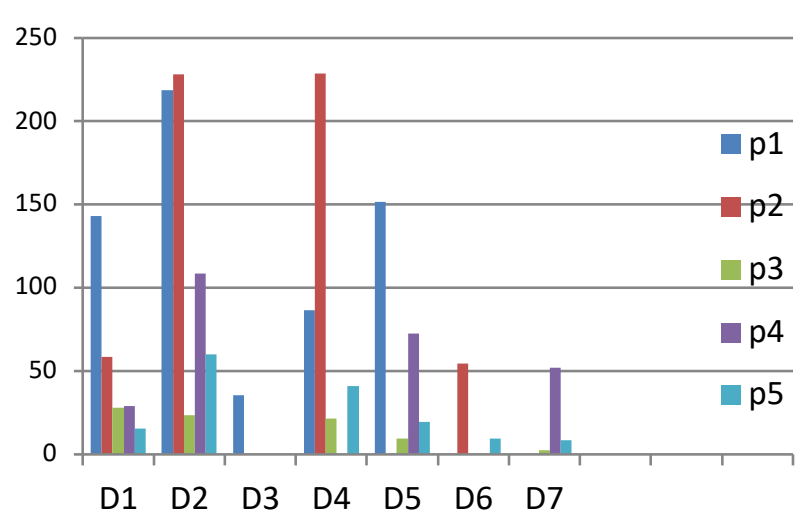

Fig. 3. Serum levels of C Reactive Proteins ( $\mathrm{mg} / \mathrm{L}$ ) in Patients during IVIg treatment.

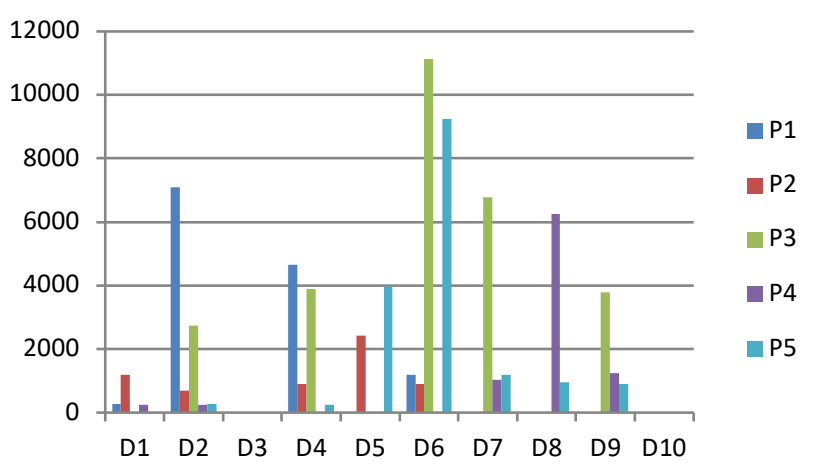

Fig. 4. Serum levels of D Dimer (ng/ml) in Patients during IVIg treatment.

6. Complications, treatment, outcomes, and duration of illness: The most common complications were ARDS and cytokine storm in all 5 patients. Bacteremia and urinary tract infection were seen in 4 patients. All 5 of them were admitted in ICU. P1was 62 years male patient and was referred from outside with COVID-19 positive status and acute respiratory distress syndrome (ARDS). He was mechanically ventilated but developed catastrophic COVID-19 infection with severe acute acrocyanosis and digital gangrene. He subsequently succumbed to his disease due to multi organ dysfunction. P2 had chronic kidney disease (CKD) and maintenance hemodialysis was done. P4 was 72 years male patient with moderate Left ventricle systolic dysfunction, CRT device in situ and a past history of pulmonary tuberculosis. His hypoxemic respiratory failure was managed with escalation of oxygen therapy i.e., High flow nasal cannula (HFNC) along with oxygen via venturi mask. He was discharged after 14 days of hospital stay. P3 and P5 were managed with HFNC.

All 5 patients were treated with antiviral therapy, oxygen inhalation, and empirical antibiotics, Anticoagulants, Dexamethasone and IVIg. P5 also received convalescent COVID plasma (CCP). P3 and P4 were given Tocilizumab as per institution's SOC. The median length of stay was 13 days with range from 8 to 17 days. Of the five patients $\mathrm{P} 1$ succumbed to catastrophic COVID-19 disease. P2 to P5 were discharged in stable clinical condition and off oxygen therapy.

\section{DISCUSSION}

This small case series of 5 patients provide evidence to support the administration of IVIg for improving clinical outcomes in severe and/or critical COVID-19 patients with severe respiratory system involvement. Intravenous 
immunoglobulin (IVIg) has been explored for the treatment COVID-19 by many investigators. Although placebocontrolled, double-blind randomized clinical trials are lacking, current data from either retrospective study, case series or open-label randomized controlled trials provide an indicator that IVIg immunotherapy could benefit severe and critically ill COVID-19 patients [4], [6], [10], [11], [16]-[18]. It is possible that IVIg could be more effective in patients with more severe immune response. The SARS-CoV-2 infection triggers massive influx of activated immune cells to the lungs causing cytokine storm and severe lung lesions. As discussed, earlier CRS is the major cause of morbidity and mortality, several drugs targeting inflammation such as steroids, inhibitors of cytokines and inflammatory markers and also immunomodulatory drugs such as neutralizing antibodies to IL-6 and GM-CSF; recombinant IL-1 receptor antagonist, blocking antibodies to IL-6 receptor and intravenous immunoglobulin have provided encouraging results [1]. While the aforementioned immunotherapy's target a specific molecule, IVIg exerts its therapeutic benefits by pleiotropic immunomodulating actions. In COVID-19 it acts through its immunomodulatory effect to suppress a hyperactive immune response (CRS) that is seen in severe and critical cases.

The COVID-19 outbreak has had a huge impact on the globe and India had been recently hit by a huge second corona virus wave. However, with much more cases of moderate and severe-critical infection in the second wave, there is a need to explore new promising treatment strategies which could salvage these severe critical ill patients. We have hereby shared our experience in such cases with IVIg during the first wave of corona virus infection in 2020. Five severely ill COVID-19 patients are described in whom standard treatments were administrated along with IVIg which prevented the deterioration of clinical symptoms in all except one (P1).

There are several reports in the literature which show that early and timely administration ( $\leq 7$ days post admission) of high-dose ( $>15 \mathrm{~g} /$ day) IVIg improves the prognosis of severe and critical-type patients with COVID-19. In a retrospective cohart study, Shao et al evaluated the efficacy of 0.1$0.5 \mathrm{~g} / \mathrm{kg} /$ day of IVIg in 325 severe and critically ill COVID19 patients. [16]. IVIg was given for a duration of 5-15 days along with standard of care measures. Although a multicentre study, it had certain limitations of being a retrospective study, usage of a wide range of IVIg dose, varying duration of treatment and that they did not analyze IVIg effects on various inflammatory cytokines and immune cells. Other studies are mostly case series or case reports. There is only study that was randomized open-label trial [4], [6], [10], [11], [17], [18]. In another retrospective study, Xie et al showed that IVIG benefited these patients if IVIg therapy is initiated early [10]. Zhou et al reported that short-term moderate-dose corticosteroid plus IVIg ( $20 \mathrm{~g} /$ day) might effectively benefit COVID-19 patients who did not respond to low-dose therapy (10 g/day) [11]. The only randomized controlled study by sakoulas et al on IVIg also combined methylprednisolone [17]. However, these authors concluded that in view of the co administration of corticosteroid, the therapeutic benefits of IVIg in itself cannot be evaluated. However, steroids were included as an SOC after this study was done and now world over steroids are recommended in moderate to severe COVID-19 patients. It is prudent to note that autoimmune and inflammatory diseases have better outcomes if IVIg is combined with steroids. Also, IVIg has steroid-sparing effects [2]. Xie et al reported beneficial effect of early administration of IVIg (within 48 hours of hospital admission) along with low dose corticosteroids for 5-7 days. They reported a reduction of use of mechanical ventilation, hospitalization and overall clinical effectiveness [10]. Zantah et al reported improved clinical outcomes in most subjects who were treated with IVIG along with subcutaneous Anakinra, a recombinant modified IL-1 receptor antagonist [18]. Non-SARS-COV-2 specific IVIG have been given AIII recommendation in NIH COVID-19 treatment guidelines. In our small retrospective case series, we have used a fixed high dose of $0.4 \mathrm{~g} / \mathrm{kg} /$ day of IVIg for a total duration of 5 days and none of the patient received $<25 \mathrm{~g}$ /day of IVIg. All five of them were initiated on IVIg within 7 days along with institute's SOC treatment. Of these 5 patients, 4 were discharged to home in stable clinical condition.

The precise mechanism by which IVIg might help these sick COVID-19 patients is not yet precisely understood. The reduction in the inflammatory mediators following IVIg therapy suggests that IVIg works through its immunomodulatory effect to suppress a hyperactive immune response rather than boosting the immune system. It targets cytokine storm by complement scavenging, inhibition of innate immune cells and effector T-cell activation, and expansion of Tregs [2]. Recently, Chang et al reported that SARS-CoV-2 encodes a super antigen motif near its S1/S2 cleavage site that might trigger cytokine storm. [20]. As IVIg contains antibodies reacting against SARS-CoV-2 antigens, IVIg might inhibit super antigen-mediated T-cell activation and cytokine release [21].

Are there any biomarkers to evaluate response of IVIg in COVID-19 patients? Do all COVID-19 patients respond to IVIg? What shall be the ideal dose and time for its administration in these patients? These are some questions which need to be answered. IVIg is no magic bullet. The current evidence from various studies discussed above suggests that early initiation of high dose IVIg therapy could benefit appropriately selected high risk COVID-19 patients. It does not benefit all patients. Therefore, studies are required to identify potential biomarkers of IVIg response in COVID19 patients. As a clinician, one should be able to analyze the trend in inflammatory markers, decide on the timing of impending cytokine storm and its timely control through immunomodulators, corticosteroids, and cytokine antagonists. The initiation of the cytokine storm takes place in the second week after initiation of symptoms and represents the time window in which immunomodulation is likely to be most beneficial [22]. Studies by Shao et al. [16] and Sakoulas et al. [17] show that IVIg decreases IL-6 levels in the plasma. Majority of the studies have also reported that C-reactive protein (CRP) is down regulated following IVIg therapy. We also studied trend of inflammatory markers including CRP, IL-6, Ferritin, LDH and D Dimer during IVIg treatment in our case series. It was observed that IVIg decreases CRP and D Dimer levels in the plasma. However, we could not find any correlation between serum levels of IL6, LDH and Ferritin with IVIg treatment. 
Nonetheless, the acute surge in inflammatory markers with deteriorating clinical condition and increase in oxygen requirement of the patient despite institution of SOC does help clinician to select patients early with impending cytokine storm and initiating IVIg. Timely and high dose IVIg initiation is the key to help treat severe and critically ill COVID-19 patients. In our case series the intravenous administration of immunoglobulin's has shown a rapid clinical improvement as evident by the short period of need for respiratory support, early clinical recovery, improvement in inflammatory markers (CRP and D Dimer) and the radiological findings.

\section{CONCLUSIONS}

The promising result from this case series is that four of the five patients survived hospital discharge. In patients of severe and critical COVID-19 who have severe pulmonary involvement with hypoxemia, treatment with IVIg led to a significant clinical improvement and concomitant reduction of serum inflammatory markers CRP and D Dimer. The crux in the successful management of these patients is the timely identification of the onset of cytokine storm, as the greatest benefit with IVIg is achieved with timely and high dose administration. Howbeit, the results are encouraging and warrants further studies.

\section{REFERENCES}

[1] R. Bonam, S. V. Kaveri, A. Sakuntabhai, L. Gilardin and J. Bayry, "Adjunct immunotherapies for the management of severely ill COVID19 Patients," Cell Reports Medicine 1(2): 100016, 2020.

[2] C. Galeotti, S. V. Kaveri and Bayry, J, "Intravenous immunoglobulin immunotherapy for coronavirus disease-19 (COVID-19)," Clinical \& Translational Immunology 9(10): e1198, 2020.

[3] J. B. Moore and C. H. June, "Cytokine release syndrome in severe COVID-19," Science 368(6490):473-474.

[4] N. Mohtadi, A. Ghaysouro, S. Shirazi, A. Sara, E. Shafiee, E. Bastani, T. Kokhazadeh and H. Tavan, "Recovery of severely ill COVID-19 patients by intravenous immunoglobulin (IVIG) treatment: A case series," Virology 548:1-5, 2020.

[5] H. Shi, C. Zhou, P. He, S. Huang, Y. Duan, X. Wang and Y. Zha, "Successful treatment with plasma exchange followed by intravenous immunoglobulin in a critically ill patient with COVID-19," International Journal of Antimicrobial Agents 105974, 2020.

[6] W. Cao, X. Liu, T. Bai, H. Fan, K. Hong, H. Song and T. Lie, "HighDose Intravenous Immunoglobulin as a Therapeutic Option for Deteriorating Patients with Coronavirus Disease 2019," Open Forum Infectious Diseases 7(3), 2020.

[7] M. Lanza, G. E. Polistina, P. Imitazione, A. Annunziata, V. Di Spirito, C. Novella and G. Fiorentino, "Successful intravenous immunoglobulin treatment in severe COVID-19 pneumonia," IDCases e00794 - e00794, 2020.

[8] K. Chiotos, H. Bassiri, E. M. Behrens, A. M. Blatz, J. Chang, C. Diorio and A. R. O. John, "Multisystem Inflammatory Syndrome in Children During the Coronavirus 2019 Pandemic: A Case Series," Journal of Pediatric Infectious Disease Society 9(3): 393-398, 2020.

[9] Z. Shao, Y. Feng, L. Zhong, Q. Xie, M. Lei, Z. Liu, C. Wang, J. Ji, L. Huiheng, Z. Gu, Z. Hu, L. Su, M. Wu and Z. Liu, "Clinical efficacy of intravenous immunoglobulin therapy in critical patients with COVID19: A multicenter retrospective cohort study," medRxiv [Online] 2020.

[10] Y. Xie, S. Cao, H. Dong, Q. Li, E. Chen, W. Zhang and R. Wang, "Effect of regular intravenous immunoglobulin therapy on prognosis of severe pneumonia in patients with COVID-19," Journal of Infection 81(2): 318-356, 2020.

[11] Z. G. Zhou, S. M. Xie, J. Zhang, F. Zheng, J. H. Liu, C. L. Cai and L. Zhang, "Short-Term Moderate-Dose Corticosteroid Plus Immunoglobulin Effectively Reverses COVID-19 Patients Who Have Failed Low-Dose Therapy," Preprints, 2020.
[12] FDA News Release: Coronavirus (COVID-19) Update: FDA Coordinates National Effort to Develop Blood-Related Therapies for COVID-19. April 3, 2020. https://www.fda.gov/news-events/pressannouncements/coronavirus-covid-19-update-fda-coordinatesnational-effort-develop-blood-related-therapies-covid-19.

[13] R. T. Gandhi, J. B. Lynch and C. del Rio, "Mild or Moderate Covid19", New England Journal of Medicine 383:1757-66, 2020.

[14] A. Agarwal, A. Sharma, R. Jakhar and M. Agarwal, "Severe Acute Acrocyanosis and Digital gangrene as a sign of Catastrophic COVID19 infection", Journal of Clinical and Diagnostic Research 15(4): OD12OD15, 2021.

[15] M. Prokop, W. van Everdingen, T. van Rees Vellinga, H. Quarles van Ufford, L. Stöger, L. Beenen, B and COVID-19 Standardized Reporting Working Group of the Dutch Radiological Society, "CORADS: A Categorical CT Assessment Scheme for Patients Suspected of Having COVID-19-Definition and Evaluation," Radiology 296(2): E97-E104, 2020.

[16] Z. Shao, Y. Feng, L. Zhong, Q. Xie, M. Lei, Z. Liu and Z. Liu, "Clinical efficacy of intravenous immunoglobulin therapy in critical ill patients with COVID-19: a multicenter retrospective cohort study", Clinical and Translational Immunology 9(10): e1192, 2021.

[17] G. Sakoulas, M. Geriak, R. Kullar, K. L. Greenwood, M. K. Habib, A. Vyas and F. Haddad, "Intravenous immunoglobulin (IVIG) significantly reduces respiratory morbidity in COVID-19 pneumonia: a prospective randomized trial", medRxiv, [Online] 2020.

[18] M. Zantah, E. D. Castillo, A. J. Gangemi, M. Patel, J. Chowdhury, S. Verga and R. Carricchio, "Anakinra and intravenous IgG versus tocilizumab in the treatment of COVID-19 pneumonia", medRxiv [Online], 2020.

[19] W. J. Wiersinga, A. Rhodes, A. C. Cheng, S. J. Peacock and H. C. Prescott, "Pathophysiology, Transmission, Diagnosis, and Treatment of Coronavirus Disease 2019 (COVID-19): A Review," JAMA 324(8): 782-793, 2020.

[20] M. H. Cheng, S. Zhang, R. A. Porritt, M. Noval Rivas, L. Paschold, E. Willscher and I. Bahar, "Superantigenic character of an insert unique to SARS-CoV-2 spike supported by skewed TCR repertoire in patients with hyperinflammation," Proceedings of the National Academy of Sciences of the United States of America 117(41): 25254-25262, 2020.

[21] J. M. Díez, C. Romero and R. Gajardo, "Currently available intravenous immunoglobulin contains antibodies reacting against severe acute respiratory syndrome coronavirus 2 antigens," Immunotherapy 12(8): 571-576, 2020.

[22] M. Buszko, J. H. Park, D. Verthelyi, R. Sen, H. A. Young and A. S. Rosenberg, "The dynamic changes in cytokine responses in COVID19: a snapshot of the current state of knowledge", National Immunology 21(10):1146-1151, 2020.

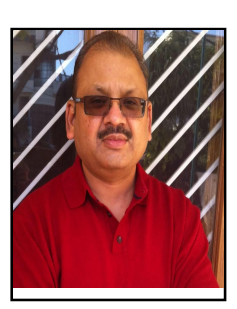

Arun Agarwal, born on December 14, 1961, in Jaipur, India completed his MBBS and MD from the prestigious SMS Medical College in Jaipur. He completed his MD in 1989 and then joined housejob at Sir Gangaram Hospital and Hindu Rao Hospital in New Delhi, India.

He later started working as an independent consultant in Internal medicine with a keen interest in tropical and critical care medicine. He has about 50 peer reviewed publication in national and international journals. He is currently heading the internal medicine department at Fortis Escorts Hospital in Jaipur and is involved in teaching DNB students enrolled in the institute.

Dr Agarwal is a member of several prestigious societies including API, CSI and ISCCM. He has been awarded Dr JC Patel best paper award in 2016 for his work on hemophagocytic lympho-histiocytosis. 